\title{
FPGA Implementation of a Chaos-based Stream Cipher and Evaluation of its Performances
}

\author{
Fethi Dridi \\ Univ. Monastir LE $\mu$ E \\ 5019 Monastir Tunisia \\ Univ. Nantes, CNRS, IETR UMR \\ 6164, F-44000 Nantes France
}

\author{
Carinelle Atamech \\ Univ. Lebanese
}

\author{
Safwan El Assad \\ Univ. Nantes, CNRS, IETR UMR \\ 6164, F-44000 Nantes France
}

\author{
Wajih Elhadj Youssef, Mohsen Machhout \\ Univ. Monastir LE $\mu E$ \\ 5019 Monastir Tunisia
}

\begin{abstract}
Chaos based stream cipher (CSC) has caught the attention of various security applications, especially for military needs and protection in Internet of Things (IoT). In fact, computing and memory resources have been suited by chaos-based stream cipher for real-time communication. In this paper, we designed a chaos-based stream cipher using a robust pseudo chaotic number generator (RPCNG). The simulation of the proposed CSC is done in VHDL using the ISE Design Suite 14.6 tool of Xilinx with finite computing precision $N=32$-bit and the hardware implementation is realized on the SAKURA-G FPGA board. The proposed system requires 6036 slices LUTs as hardware cost and achieves throughput of 301.184 Mbps. It is robust against statistical attacks and thus can be used in all applications that require confidentiality.
\end{abstract}

\section{Introduction}

The transfer of multimedia data (texts, images, videos, etc.) is growing exponentially and requires reliable techniques to ensure the confidentiality, integrity and authenticity of the data. Data encryption and steganography are the techniques used to achieve confidentiality. The steganography technique is only used when the encryption technique is prohibited (for example in the case of dictatorships in certain countries). In addition, steganography allows dissimulating only small, secret messages compared to the size of the cover medium. Thus, for huge data it is necessary to use symmetric encryption techniques such as block or stream cipher. Block ciphers operate on fixed blocks transformation of plaintext and allows various modes of encryption. Generally, they are more secure then stream ciphers, however, they are more complex in software and hardware implementation and thus they have less computational performance compared to stream ciphers. Stream ciphers operate continuously on clear text sequences (sample by sample) so they are more suitable for communication data and have better computational complexity compared to block ciphers.

Consequently, in September 2008, the eSTREAM project finally proposed seven stream ciphers, which are considered to be secure and fast. They are practically used as standard of stream ciphers [1]. Alternatively, in the literature, several works have proposed stream encryption based on chaotic systems implemented by software [2], [3], [4]. Indeed, such cryptosystems have a strong nonlinearity compared to standard cryptography and intrinsically there is randomness in the produced chaotic sequences. The chaotic signal is presented as noise but is fully reproducible due to the deterministic mathematical model used. Such cryptosystems can provide a good combination of high security and computational performance. Furthermore, the designing of chaos-based cryptosystems is easier than designing standard cryptosystems.

In this paper, we propose and realize an effective hardware implementation on a FPGA board of a stream cipher based on Robust Pseudo-Chaotic Numbers Generator (RPCNG). The results obtained by the proposed system are compared to some published works [5], [6], [7]. The structure of the paper is arranged as follows: section 2 describes the design and the hardware implementation of the proposed chaos-based stream cipher as well as its hardware metrics performance; section 3 provides the security analysis of the system and section 4 summarizes the whole paper. 


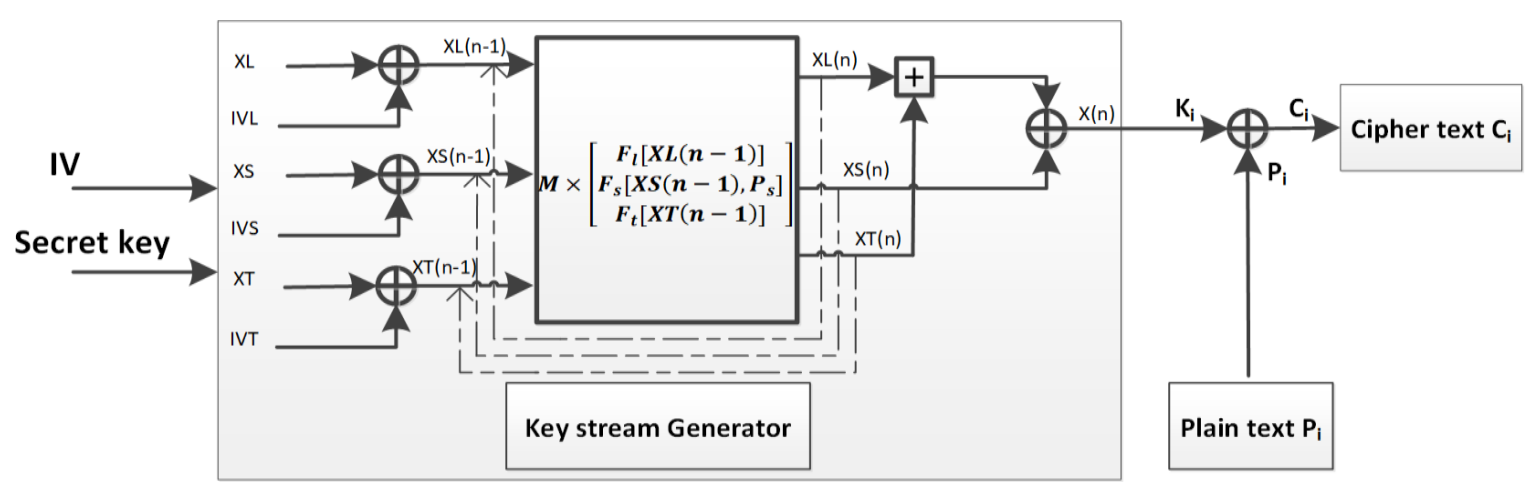

Figure 1. Block diagram of stream cipher

\section{Hardware implementation of the proposed CSC}

The architecture of the proposed chaos-based stream cipher is presented in Figure 1.

The key stream $K_{i}$ produced by the chaotic system is xored with the plaintext $P_{i}$ (any kind of data: text, image, video to protect) for generating the cipher text $C_{i}$ (data protected) as showed by equation 1:

$$
C_{i}=P_{i} \oplus K_{i}
$$

\subsection{Description of the chaotic key stream generator}

The proposed key stream generator of Figure 1 is an improved architecture compared to those given in our previous works [8] and [9]. In this architecture, in addition of the discrete Logistic and Skew-Tent map we integrate the discrete 3-D Chebyshev map. The 3-D Chebyshev map allows greater robustness against algebraic attack. The three discrete chaotic maps are weakly coupled and the key stream is generated by combination of the three-coupled outputs. The secret key $K$ of the system is constituted by:

- The initial conditions $X L, X S$ and $X T$ of the three chaotic maps: Logistic, Skew-Tent and 3-D Chebyshev map respectively, each ranging from 1 to $2^{N}-1$.

- The control parameter $P_{S}$ of Skew-Tent map, in the range $[1 ; 2 N-1]$ and the coupling parameters $\epsilon_{i j}$, ranging from $[1 ; 2 k]$ and $k \leq 5$.
The initial values $X L(0), X S(0)$ and $X T(0)$ of the used chaotic maps are calculated as follows:

$$
\left\{\begin{array}{l}
X L(0)=X L \oplus I V L \\
X S(0)=X S \oplus I V S \\
X T(0)=X T \oplus I V T
\end{array}\right.
$$

Where IVL, IVS and IVT are provided by the initial value $I V$. The output samples $X L(n), X S(n)$ and $X T I(n)$ of the coupled matrix $M$ are used to produce the output sequence $X(n)$ as showed in Figure 1 . The internal system is governed by the following equation:

$$
\left[\begin{array}{l}
X L(n) \\
X S(n) \\
X T(n)
\end{array}\right]=M \times\left[\begin{array}{c}
F_{l}[X L(n-1)] \\
F_{s}\left[X S(n-1), P_{s}\right] \\
F_{t}[X T(n-1)]
\end{array}\right]
$$

Where $M$ represent the weak coupling matrix and given by:

$$
M=\left[\begin{array}{ccc}
M_{11} & \epsilon_{12} & \epsilon_{13} \\
\epsilon_{21} & M_{22} & \epsilon_{23} \\
\epsilon_{31} & \epsilon_{32} & M_{33}
\end{array}\right]
$$

With $\quad M_{11}=\left(2^{N}-\epsilon_{12}-\epsilon_{13}\right), \quad M_{22}=$ $\left(2^{N}-\epsilon_{21}-\epsilon_{23}\right), \quad M_{33}=\left(2^{N}-\epsilon_{31}-\epsilon_{32}\right), \quad$ and $F_{l}[X L(n-1)], \quad F_{s}\left[X S(n-1), P_{s}\right]$, and $F_{t}[X T(n-$ 1)] are the three nonlinear chaotic functions producing samples at instant $n$ from samples at instant $(n-1)$. They are respectively defined as follows:

Discrete Logistic map:

$$
X L(n)=F_{l}[X L(n-1)]=\left\{\begin{array}{cc}
{\left[\frac{X L(n-1)\left[2^{N}-X L(n-1)\right]}{2^{N-2}}\right]} & \text { if } X L(n-1) \neq\left[3 \times 2^{N-2}, 2^{N}\right] \\
2^{N}-1 & \text { otherwise }
\end{array}\right.
$$


Discrete Skew-Tent map:

$$
X S(n)=F_{S}\left[X S(n-1), P_{S}\right]=\left\{\begin{array}{cc}
\left\lfloor\frac{2^{N} \times X S(n-1)}{P_{S}}\right\rfloor & \text { if } 0<X S(n-1)<P_{S} \\
\left\lfloor 2^{N} \times \frac{\left[2^{N}-X S(n-1)\right]}{2^{N}-P_{S}}\right] & \text { if } P_{S}<X S(n-1)<2^{N} \\
2^{N}-1 & \text { otherwise }
\end{array}\right.
$$

Discrete 3-D Chebyshev map:

$$
X T(n)=F_{t}[X T(n-1)]=\left\lfloor 2^{(-2 N+2)} \times\left(4 \times\left(X T-2^{(N-1)}\right)^{3}-3 \times 2^{(2 N-2)} \times\left(X T-2^{(N-1)}\right)\right)+2^{(N-1)}\right\rfloor
$$

We present below the main statistical properties (histogram uniformity and NIST test) of each chaotic map used alone: Logistic, Skew-Tent and 3-D Chebyshev map, as base of the proposed chaos based stream ciphers. The chaotic maps are discretized making them run over a finite precision $N=32$.

First, we have plotted in Figures 2, 3, and 4 the histograms of the generated sequences $X L(n)$, $X S(n)$, and $X T(n)$ respectively. Visually, the generated sequences are not uniform over all the values $\left[1,2^{32}-1\right]$.

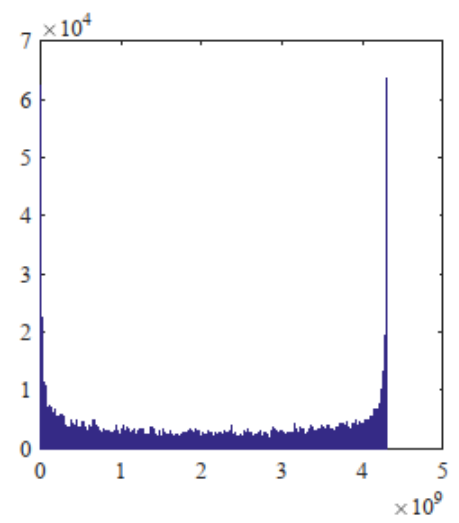

Figure 2. Histogram of the sequence $X L(n)$ generated by the discrete Logistic map

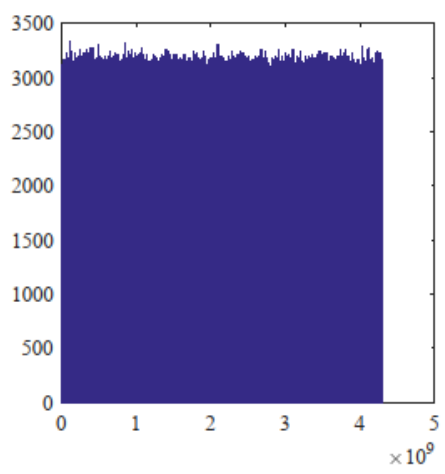

Figure 3. Histogram of the sequence $X S(n)$ generated by the discrete Skew-Tent map

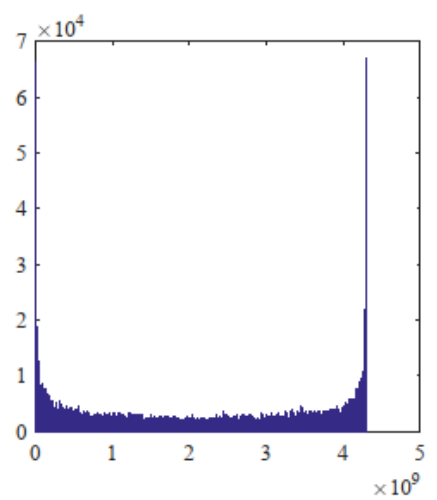

Figure 4. Histogram of the sequence $X T(n)$ generated by the discrete 3-D Chebyshev map

We confirm the non-uniformity of these sequences using the Chi-square test. The experimental Chi-square $\chi^{2}$ value is given by:

$$
\chi_{\exp }^{2}=\sum_{i=0}^{N-1} \frac{\left(O_{i}-E_{i}\right)^{2}}{E_{i}}
$$

Where $N=1000$ is the number of classes, $O_{i}$ is the number of calculated samples in the ith class $E_{i}$, and $E_{i}=10^{8} / N$ is the expected number of samples of a uniform distribution.

Table 1, gives the results obtained of the Chisquare test. The calculated experimental value $\chi_{\text {exp }}^{2}$ is higher than the theoretical one $\chi_{t h}^{2}$. This asserts the non-uniformity of the histograms of sequences $X L(n), X S(n)$, and $X T(n)$.

Table 1. Chi-square test

\begin{tabular}{|l|c|c|c|}
\hline \multicolumn{1}{|c|}{$\begin{array}{c}\text { Chi-Square test } \\
(\mathbf{1 0 0 0 , 0 . 0 5 )}\end{array}$} & $\chi_{\text {th }}^{2}$ & $\chi_{\text {exp }}^{2}$ & Result \\
\hline Logistic map & 1073.64 & 3990034.96 & $\begin{array}{c}\text { not } \\
\text { uniform }\end{array}$ \\
\hline Skew-Tent map & 1073.64 & 1171.35 & $\begin{array}{c}\text { not } \\
\text { uniform }\end{array}$ \\
\hline 3-D Chebyshev map & 1073.64 & 4234181.41 & $\begin{array}{c}\text { not } \\
\text { uniform }\end{array}$ \\
\hline
\end{tabular}


Second, we apply the NIST statistical test by generating 100 different sequences, each of size equal to 3,125,000 samples and using different secret key for each sequence. In Figure 5, 6, and 7 we present the results obtained of the NIST test.

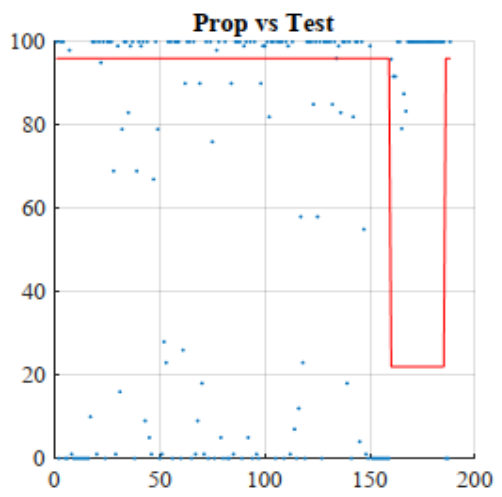

Figure 5. NIST test results of the Logistic map

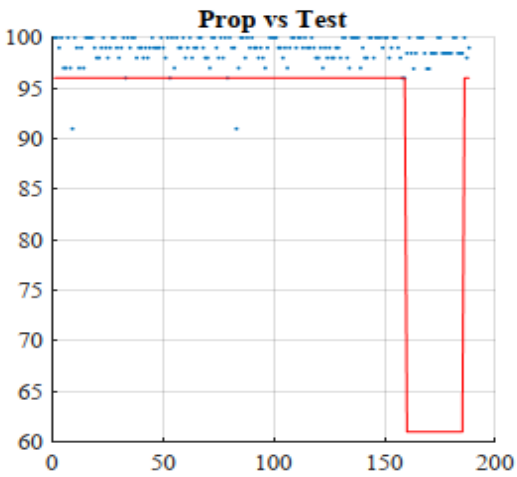

Figure 6. NIST test results of the Skew-Tent map

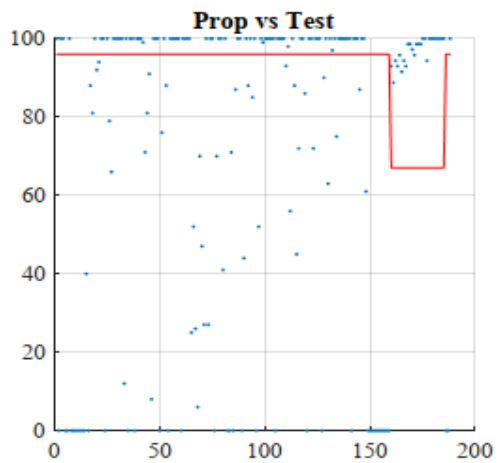

Figure 7. NIST test results of the 3-D Chebyshev map

Obtained results show that sequences $X L(n)$, $X S(n)$, and $X T(n)$ does not pass all the NIST tests. Also, we remark that the Skew-Tent map has better NIST performance than the other studied chaotic maps: Logistic and 3-D Chebyshev map.

\subsection{Hardware implementation and evaluation of the proposed CSC}

The design was coded in hardware descriptive language (VHDL). Units such as dividers, multipliers, subtractors and adders in fixed point arithmetic representation are used in FPGA-based implementation of the proposed stream cipher. The multiplication and division processes are a bit more complex and generally they require a much more complex logic circuits. For this reason, we try to avoid their use whenever possible, unless there are special cases. Therefore, to achieve an efficient division or multiplication by $2^{n}$, we apply the following known rule: in binary representation shifting to the right of $n$ positions corresponds to a division by $2^{n}$ and shifting to the left of $n$ positions corresponds to a multiplication by $2^{n}$. For the implementation of the proposed stream cipher, we used the ISE Design Suite 14.6 tool of Xilinx. This tool constitutes an efficient design environment, which brings together all the necessary mechanisms for the implementation of a digital circuit, ranging from behavioral description in VHDL to generation of the corresponding diagram in logic gates. Table 2 summarizes the hardware resources occupied by the proposed CSC following the synthesis and implementation processes.

The implementation uses 272 slice registers, 6,036 slice Look Up Tables (LUTs) and 27 DSP blocks. It produces one sample at each clock cycle and the throughput obtained is $301.184 \mathrm{Mbps}$ for a clock frequency of $9.412 \mathrm{MHz}$. Table 3 provides a summary of the frequency, throughput and resource uses for the implementation of the proposed encryption algorithm, also a comparative result obtained by some published stream ciphers and implemented on FPGA platforms.

Table 2. Usage statistics for xilinx spartan6 xc6slx75-2-csg484 chip of the proposed FPGA based stream cipher

\begin{tabular}{|l|c|}
\hline \multicolumn{2}{|c|}{ Device utilization summary } \\
\hline \multicolumn{1}{|c|}{ Logic Utilization } & Used / utilization \% \\
\hline Number of Slice Registers & $272 / 1$ \\
\hline Number of Slice LUTs & $6036 / 12$ \\
\hline Number of occupied Slices & $1733 / 14$ \\
\hline Number of bonded IOBs & $21 / 6$ \\
\hline Number of DSP48A1s & $27 / 20$ \\
\hline
\end{tabular}

From the results given in Table 3, we can notice that the proposed CSC has satisfactory performance compared to the other mentioned hardware implementations of stream ciphers. However, this comparison remains relative, since the hardware implementations considered are not made all on the same FPGA platform. 
Table 3. Comparison of hardware metrics with some published works

\begin{tabular}{|l|c|c|c|c|}
\hline \multirow{2}{*}{$\begin{array}{l}\text { Hardware } \\
\text { metrics }\end{array}$} & \multicolumn{4}{|c|}{ Ref } \\
\cline { 2 - 5 } & {$[5]$} & {$[6]$} & {$[7]$} & Proposed \\
\hline LUTs & 2363 & 2718 & 1169 & 6036 \\
\hline FFs & - & 791 & 416 & 231 \\
\hline $\begin{array}{l}\text { Frequency } \\
\text { (MHz) }\end{array}$ & 18.5 & 15.598 & 89.27 & 9.412 \\
\hline $\begin{array}{l}\text { Throughput } \\
\text { (Mbps) }\end{array}$ & 565 & 124 & 1178 & 301.184 \\
\hline
\end{tabular}

\section{Security analysis of the proposed CSC}

In what follows, we present the key space and statistical analysis obtained from the proposed chaotic system.

\subsection{Key space analysis}

The size of the secret key of a given cryptosystem is the total of initial conditions and parameters of the cryptosystem. Since, the size of the secret key is linked directly to the security level of a symmetric cryptosystem, it is recommended to use secret keys of size typically larger than 128 bits to resist against brute force attacks. Indeed, the integration of three chaotic maps in the proposed cryptosystem allows, on the one hand, to increase the size of the secret key and, on the other hand, to improve the quality of the generated sequences. The size of the secret key of the proposed stream cipher is given by:

$$
K=X L+X S+P_{s}+X T+\left(6 \times \epsilon_{i j}\right)=158 \text { bits }
$$

Where the size of $X L, X S, X T$ and $P_{S}$ is equal to 32 bits each and the size of $\epsilon_{i j}$ is equal to 5 bits. The key space of the secret key is $2^{158}$ different combinations. Therefore, the size of the secret key used for image encryption is large enough to make brute force attack impossible.

\subsection{Statistical Analysis}

The majority of statistical attacks against stream cipher algorithms aim to detect and exploit the weaknesses linked to the key stream generators used, through the statistical analysis on the key stream sequences $K_{i}$ and cipher sequences $C_{i}$. In this regard, firstly we used the standard NIST SP 800-22 tests [10] to evaluate the statistical properties of the proposed PCNG. All the tests are applied to $100 \mathrm{key}$ stream sequences $K_{i}$ of a total size equal to $10^{8}$ bits, generated from 100 secret keys. The results show in Table 4 indicate that the sequences produced by the proposed generator pass all the statistical tests with success. This means that the proposed PCNG produces pseudo random sequences.

Secondly, we tested the confusion property on five ciphered images, whose plain images are of different natures. The results obtained on the one hand, on the ciphered images given in Figures 8 to $12 \mathrm{c}$ and their histograms presented in Figures 8 to $12 \mathrm{~d}$ and on the other hand, the entropy and correlation measurements given in Table 6 indicate the good level of confusion.

Indeed, as we can see, from Figures 8 to $12 \mathrm{~d}$ that the histograms of the encrypted images visually seem to be distributed uniformly compared to the histograms of the plain images showed in Figures 8 to $12 \mathrm{~b}$.

To assert the uniformity of the encrypted image histograms, we apply the chi-square test. As we can see from Table 5, the experimental chi-square values are smaller than the theoretical ones, confirming thus the uniformity.

Furthermore, it is clear from Table 6 that, for the encrypted images, the information entropy is close to the optimal value 8 and the correlation coefficient is close to zero, which proves that there is no correlation between the plain and ciphered images. Whereas for the plain images, the correlation coefficient is close to one, which indicates that, the pixels are highly correlated. Moreover, these results are confirmed in Figures 13-14 that shows the correlation of horizontally, vertically and diagonally adjacent pixels in the plain and ciphered Cameraman and Pepper images. Similar results are obtained for Flowers, Gold hill and Kiel images.

Therefore, there is no similarity between the original and encrypted images.

Table 4. P-values and proportion results of nist test for the proposed pcng

\begin{tabular}{|l|c|c|}
\hline \multicolumn{1}{|c|}{ Test } & P-value & Proportion \% \\
\hline Frequency test & 0.367 & 99 \\
\hline Block-frequency test & 0.699 & 100 \\
\hline Cumulative-sums test (2) & 0.768 & 99 \\
\hline Runs test & 0.367 & 100 \\
\hline Longest-run test & 0.740 & 100 \\
\hline Rank test & 0.616 & 99 \\
\hline FFT test & 0.122 & 98 \\
\hline Non-periodic-templates (148) & 0.514 & 98.980 \\
\hline Overlapping-templates & 0.936 & 97 \\
\hline Universal & 0.514 & 98 \\
\hline Approximate Entropy & 0.367 & 100 \\
\hline Random-excursions (8) & 0.386 & 98.785 \\
\hline Random-excursion-variant (18) & 0.527 & 97.994 \\
\hline Serial test (2) & 0.526 & 99.5 \\
\hline Linear-complexity & 0.262 & 100 \\
\hline
\end{tabular}

Table 5. Theoretical and experimental values for the chi-square test

\begin{tabular}{|c|c|c|}
\hline $\begin{array}{c}\text { Chi-Square test (256, } \\
\text { 0.05) }\end{array}$ & $\chi_{t h}^{2}$ & $\chi_{\exp }^{2}$ \\
\hline
\end{tabular}




\begin{tabular}{|l|l|l|}
\hline Cameraman & 293.24 & 266.68 \\
\hline Pepper & 293.24 & 231.84 \\
\hline Flowers & 293.24 & 253.28 \\
\hline
\end{tabular}

\begin{tabular}{|l|l|l|}
\hline Gold hill & 293.24 & 221.64 \\
\hline Kiel & 293.24 & 218.18 \\
\hline
\end{tabular}

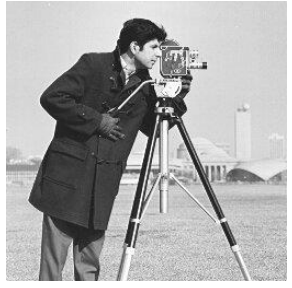

(a)

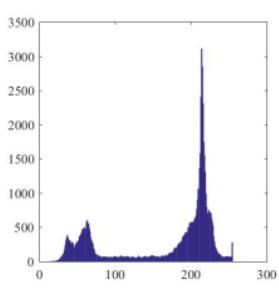

(b)

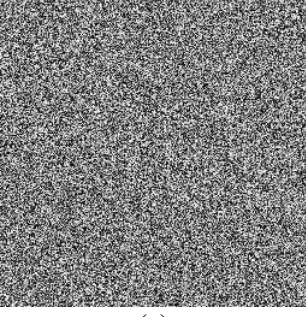

(c)

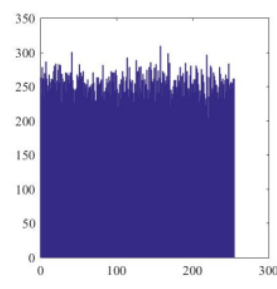

(d)

Figure 8. Result of Cameraman image. (a) Plain image, (b) Histogram of plain image, (c) Encrypted image, and (d) Histogram of encrypted image.

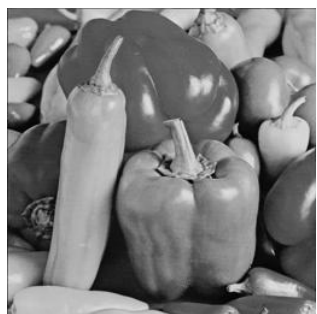

(a)

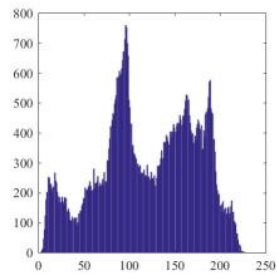

(b)

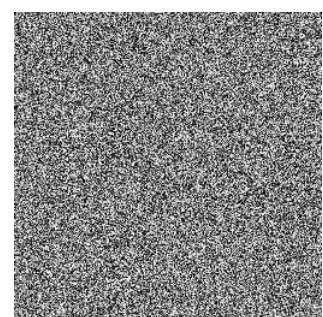

(c)

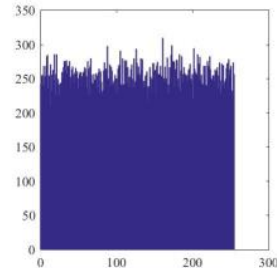

(d)

Figure 9. Result of Pepper image. (a) Plain image, (b) Histogram of plain image, (c) Encrypted image, and (d) Histogram of encrypted image.

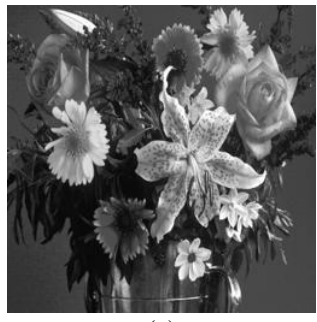

(a)

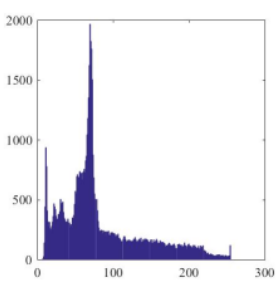

(b)

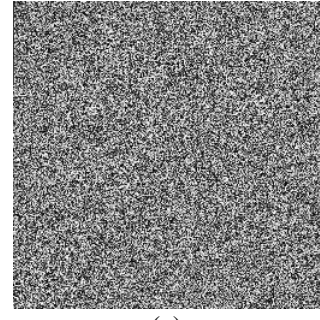

(c)

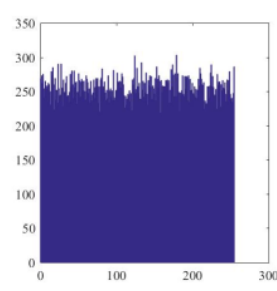

(d)

Figure 10. Result of Flowers image. (a) Plain image, (b) Histogram of plain image, (c) Encrypted image, and (d) Histogram of encrypted image.

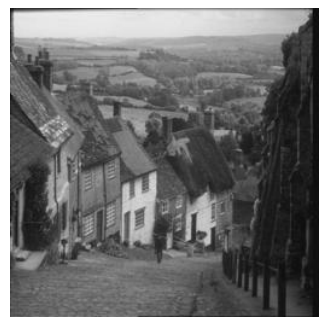

(a)

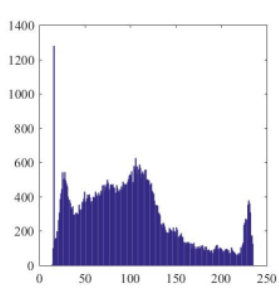

(b)

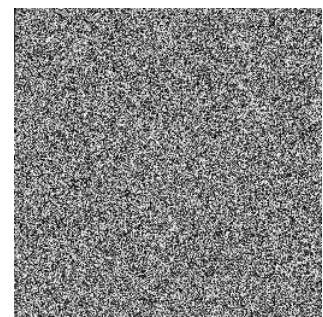

(c)

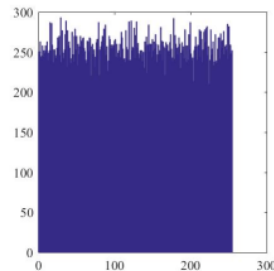

(d)

Figure 11.Result of Gold hill image. (a) Plain image, (b) Histogram of plain image, (c) Encrypted image, and (d) Histogram of encrypted image.

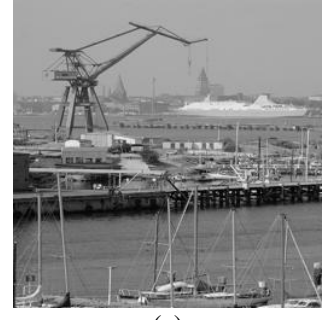

(a)

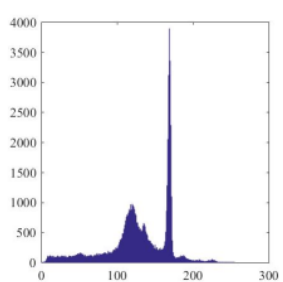

(b)

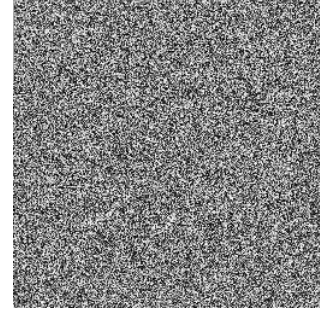

(c)

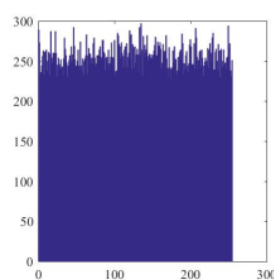

(d)

Figure 12.Result of Kiel image. (a) Plain image, (b) Histogram of plain image, (c) Encrypted image, and (d) Histogram of encrypted image. 


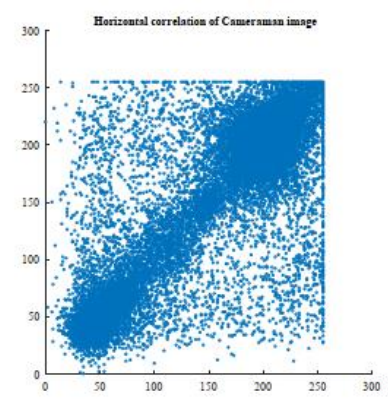

(a)

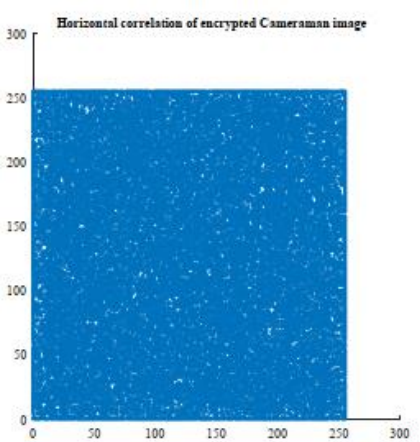

(d)

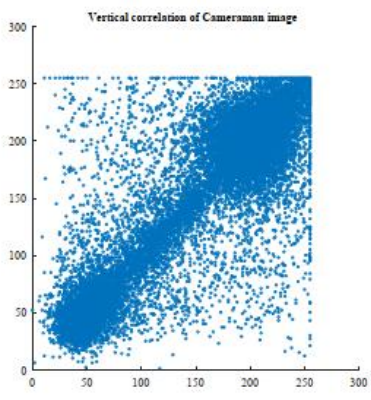

(b)

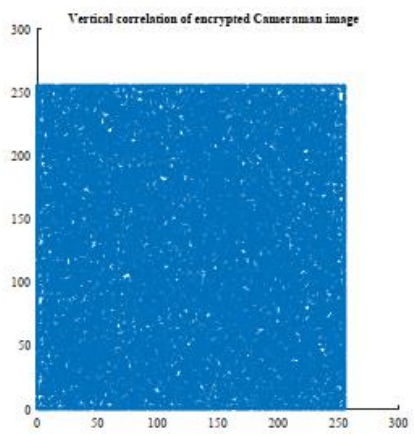

(e)

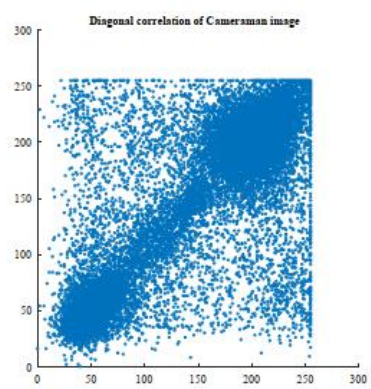

(c)

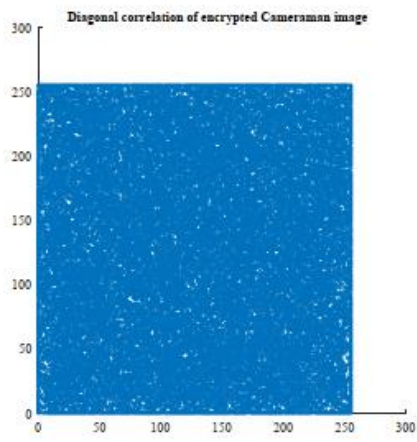

(f)

Figure 13. Correlation of adjacent pixels for Cameraman and encrypted Cameraman image in horizontal, vertical and diagonal direction

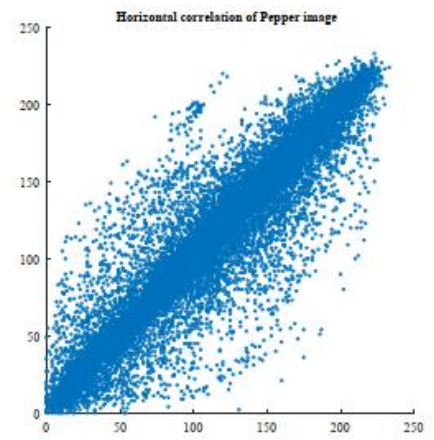

(a)

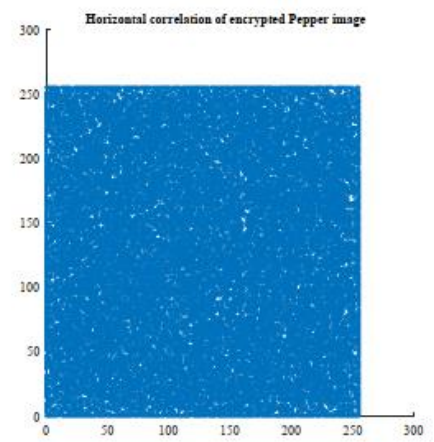

(d)

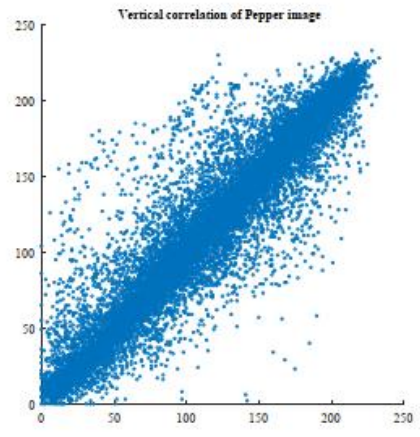

(b)

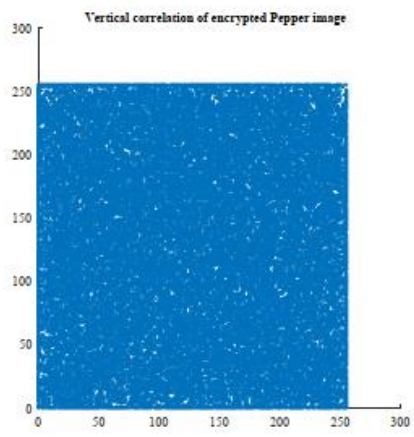

(e)

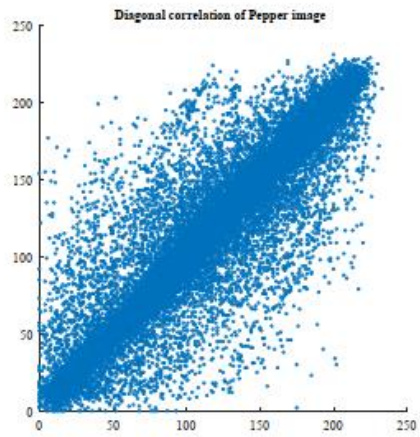

(c)

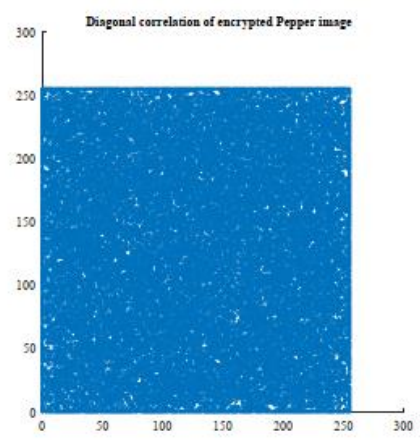

(f)

Figure 14. Correlation of adjacent pixels for Pepper and encrypted Pepper image in horizontal, vertical and diagonal direction. 


\section{Conclusion}

In summary, we have shown and commented on the various results obtained related to the hardware implementation of the proposed chaos-based stream cipher that we implemented on a Spartan-6 xc6slx752-csg484 FPGA, using the VHDL description. From these results, we can conclude that the proposed algorithm resists against statistical and brute force attack. In addition, it has a satisfactory performance in terms of hardware metrics.

Table 6. Entropy analysis and correlation coefficient values of the plain and ciphered images

\begin{tabular}{|l|c|c|c|c|}
\hline \multirow{1}{*}{\multicolumn{1}{c|}{$\begin{array}{c}\text { Test } \\
\text { image }\end{array}$}} & Entropy & \multicolumn{3}{c|}{ Correlation coefficient } \\
\cline { 3 - 5 } & & Horizontal & Vertical & Diagonal \\
\hline Cameraman & 7.0014 & 0.9024 & 0.9360 & 0.8876 \\
\hline $\begin{array}{l}\text { Cameraman } \\
\text { encrypted }\end{array}$ & 7.9550 & 0.0011 & -0.0090 & 0.0016 \\
\hline Pepper & 7.5939 & 0.9599 & 0.9679 & 0.9404 \\
\hline $\begin{array}{l}\text { Pepper } \\
\text { encrypted }\end{array}$ & 7.9580 & -0.0028 & -0.0059 & 0.0015 \\
\hline Flowers & 7.3710 & 0.9236 & 0.9493 & 0.8935 \\
\hline $\begin{array}{l}\text { Flowers } \\
\text { encrypted }\end{array}$ & 7.9555 & -0.0002 & -0.0029 & 0.0011 \\
\hline Gold hill & 7.5678 & 0.9703 & 0.9722 & 0.9530 \\
\hline $\begin{array}{l}\text { Gold hill } \\
\text { encrypted }\end{array}$ & 7.9539 & -0.0051 & -0.0002 & -0.0002 \\
\hline Kiel & 6.8502 & 0.9183 & 0.8861 & 0.8369 \\
\hline Kiel encrypted & 7.9568 & -0.0012 & -0.0019 & -0.0003 \\
\hline
\end{tabular}

\section{References}

[1] eSTREAM (2012, Mar) The eSTREAM portfolio page. [Online]. Available: https://www.ecrypt.eu.org/stream/ (Access Date: 12 September, 2018).

[2] L. Ding, C. Liu, Y.Zhang and Q. Ding, "A new lightweight stream cipher based on chaos," in Multidisciplinary Digital Publishing Institute. Symmetry, 2019, vol. 11, pp. 853.

[3] G. Vidal, R. Becheikh, R. Rhouma, and S. Belghith, "A commercial application of a chaos-based-stream cipher: Performance and Security analysis," in : 2016 11th International Conference for Internet Technology and Secured Transactions (ICITST). IEEE, 2016. pp. 39-44.

[4] P. Li, Z. Li, W. A Halang and G. Chen, "A stream cipher based on a spatiotemporal chaotic system," in Chaos, Solitons \& Fractals. Elsevier, 2007, vol. 32, no 5, pp. 1867-1876.

[5] G. Gautier, M. Le Glatin, S. El Assad, W. Hamidouche, O. Déforges, S. Guilley and A. Facon, "Hardware Implementation of Lightweight Chaos-Based Stream Cipher,” 2019.

[6] C. Tanougast, "Hardware implementation of chaos based cipher: Design of embedded systems for security applications," in Chaos-Based Cryptography. Springer, Berlin, Heidelberg, 2011. pp. 297-330.

[7] T. Bonny, R. Al Debsi, S. Majzoub and A. S Elwakil, "ardware optimized fpga implementations of high-speed true random bit generators based on switching-type chaotic oscillators," in Circuits, Systems, and Signal Processing. Springer, 2019, vol. 38, no 3, pp. 1342-1359.
[8] F. Dridi, S. El Assad, W. E Youssef and M. Machhout, "FPGA Implementation of a Pseudo-Chaotic Number Generator and Evaluation of its Performance," in : 2019 International Conference on Internet of Things, Embedded Systems and Communications (IINTEC). IEEE, 2019. pp. 231-234.

[9] O. Jallouli, S. El Assad, M. Chetto and R. Lozi, "Design and analysis of two stream ciphers based on chaotic coupling and multiplexing techniques," in Multimedia tools and applications. Springer, 2018, vol. 77, no 11, pp. 13391-13417.

[10] A. Rukhin, J. Soto, J. Nechvatal, M. Smid and E. Barker, “A statistical test suite for random and pseudorandom number genera tors for cryptographic applications," in Booz-allen and hamilton inc mclean va, 2011. 